\title{
Reporting of occupational exposures to blood and body fluids in the primary dental care setting in Scotland: an evaluation of current practice and attitudes
}

IN BRIEF
- Stresses members of the dental team are
at risk of exposure to blood borne viruses
such as HIV and Hepatitis $C$ in their
everyday working lives and an awareness
of such risks is vital for the prevention of
infection.
- Suggests that while dentists generally
have positive beliefs about reporting
occupational exposures, there are gaps in
practice.

\author{
P. Leavy, ${ }^{* 1}$ A. Templeton, ${ }^{2}$ L. Young ${ }^{3}$ and C. McDonnell ${ }^{4}$
}

\begin{abstract}
Objectives To evaluate experience, practice and beliefs regarding occupational exposures to blood and oral fluids among a random sample of 300 dentists working in Scotland's NHS primary dental services. Method A cross-sectional postal survey assessed occupational exposure policies and procedures, recent occupational exposure incidence and current management. Beliefs were measured using constructs from the theory of planned behaviour, shown to influence behaviour in this population. Results Forty-two percent of dentists responded. Fourteen percent had sustained an occupational exposure in the previous 12 months; of those, 35\% did not report their exposure. All respondents' practices had protocols in place for managing and reporting dental team member sharps injuries. Most (82\%) had protocols for mucocutaneous exposures. Less than half (48\%) had a protocol for managing and reporting patient exposures to blood or saliva. Dentists placed significantly more importance ( $z$-score $-4.44, p$ value $<0.001$ ) and necessity ( $z$-score $-4.17, p$ value $<0.001$ ) on reporting patient exposure than dentist occupational exposure. Conclusion This study suggests that while dentists generally have positive beliefs about reporting occupational exposures, there are gaps in practice.
\end{abstract}

\section{BACKGROUND}

Every time a dentist takes an instrument to a patient's mouth they are potentially putting themselves at risk of being exposed to many blood-borne viruses including Hepatitis B, Hepatitis $\mathrm{C}$ and the Human Immunodeficiency Virus (HIV). ${ }^{1}$ Transmission of these pathogens can occur following an injury sustained from a contaminated instrument such as a needle that had previously been used on a patient. There is also a risk that a dentist carrying a blood borne virus could pass that infection to a patient. ${ }^{2}$

Exposure prone procedures are defined by the UK Department of Health as invasive procedures with a risk of injury to the worker

\footnotetext{
Dental Officer and Scottish Dental Postgraduate Remote and Rural Fellow, Abban Street Dental Clinic, 22A Abban Street, Inverness, IV3 8HH; ${ }^{2}$ Research Fellow, Translation Research in a Dental Setting (TRiaDS) ${ }^{3}$ Research and Development Manager, Scottish Dental Clinical Effectiveness Programme (SDCEP), Dundee Dental Education Centre, Frankland Building, Smalls Wynd, Dundee, DD1 4HN; ${ }^{4}$ Consultant Occupational Health Physician, HSE Mid-Western Regional Hospital, Dooradoyle, County Limerick, Ireland

${ }^{*}$ Correspondence to: Paul Leavy

Email: paulleavy@ymail.com
}

Online article number E7

Refereed Paper - accepted 20 May 2014

DOI: $10.1038 /$ sj.bdj.2014.734

${ }^{\circ}$ British Dental Journal 2014; 217 : E7 or patient that could involve exposure of the patient's open tissues to the blood of the worker or vice versa. Exposure prone procedures include those where the worker's gloved hands may be in contact with sharp instruments, needle tips or sharp tissues (for example, spicules of bone or teeth) inside a patient's open body cavity, wound or confined anatomical space. ${ }^{3}$ The majority of procedures carried out by dentists, dental hygienists, and dental therapists are exposure prone..$^{4-6}$ Work flow for dental nurses including the cleaning, decontamination and sterilisation of dental instruments routinely involves risk of exposure. ${ }^{6,7}$ Occupational risk of exposure for all dental team members necessitates awareness and safe practice to minimise the chance of occupational exposure for either dental team members or patients.

The risks of being exposed to a blood borne virus are dependent upon its prevalence, communicability, and the immunologic status of the exposed individual. Hepatitis B virus (HBV) is highly contagious and most readily transmitted through percutaneous exposure to infected blood, for example puncture of the skin by a contaminated needle or scalpel. The risk of infection following a sharps injury can be as high as $30 \% .{ }^{1}$ It is estimated that in the UK
180,000 people or $0.3 \%$ of the population live with chronic Hepatitis B. ${ }^{8}$

Since its discovery in 1989, Hepatitis C (HCV) has become a major health concern worldwide. In the UK, it is estimated that 215,000 people are chronically infected with HCV. ${ }^{9}$ In Scotland the figure sits at an estimated 38,000 people or $0.7 \%$ of the population. ${ }^{9}$ The risk of contracting $\mathrm{HCV}$ following a needle-stick injury is generally regarded as being just under $2 \% .^{1}$ There is no vaccine to protect against HCV.

The incidence of HIV continues to increase across the UK. ${ }^{10}$ According to the Health Protection Agency (HPA), there were an estimated 98,400 people infected with HIV living in the UK in 2012. ${ }^{10}$ The risk of contracting HIV through a work-related sharps injury where there is exposure of the percutaneous tissues to infected blood is $0.3 \%$ or 3 per 1,000 injuries. ${ }^{11}$ The risk is lower for mucocutaneous exposures at less than 1 in $1,000^{11}$ and is negligible for exposure of infected blood to intact skin. ${ }^{12}$ There is a greater risk of contracting HIV through a sharps injury if it is a deep puncture, if the source patient was in the terminal stages of AIDS, or if the causative instrument was visibly covered in blood and punctured a large blood vessel. ${ }^{11}$ 
There is limited evidence to help quantify the most common injuries in general dental practice in Scotland. The Health Protection Agency's 2012 report Eye of the needle: United Kingdom surveillance of significant occupational exposures to bloodborne viruses in healthcare workers found that between 2002 and 2011, the majority (>90\%) of reported occupational exposures that occurred among dentists and dental nurses were sharps injuries involving hollow bore or solid needles or 'other' sharps. ${ }^{13} \mathrm{~A}$ smaller percentage $(<10 \%)$ of exposures were mucocutaneous or involved bites, scratches or unknown percutaneous sharps injuries. ${ }^{13}$ This report does not specify whether or not these OEs occurred in the primary or secondary dental care settings.

The UK dental school study by Stewardson et al. ${ }^{14}$ examined occupational exposures across three undergraduate years and found that the most commonly sustained injuries were percutaneous in nature followed by mucocutaneous aerosol splashes. ${ }^{14}$ Almost half $(46 \%)$ of all occupational exposures among third years were percutaneous, with $66 \%$ and $75 \%$ being the figures for fourth and fifth years respectively. The most common types of procedures during which students sustained an injury were administration of local anaesthesia, periodontal scaling and root planing, tidying up of instruments post-operatively (excluding needle disposal), followed by other procedures and those procedures involving a handpiece. These findings echo those from a study carried out in the US between 1987 and 1997 among staff and students at the New York University College of Dentistry, which showed that 98\% of exposures were percutaneous with the remaining being splashes to the eye. ${ }^{15}$ The most common types of instruments involved were hollow bore needles (37\%), periodontal instruments (24\%), miscellaneous (22\%), and restorative/endodontic instruments (15\%). ${ }^{15}$ The reports states that $40 \%$ of exposures occurred during treatments while the majority occurred post-operatively, eg during clean-up and recapping of needles. ${ }^{15}$ Another US study using occupational compensation claims for percutaneous injuries found the majority occurred in primary care settings (97\%) and tended to happen to dental assistants (75\%) via syringes (87\%). ${ }^{6}$ Of the 924 claims examined, only $6 \%$ were for dentists and were predominantly (82\%) syringe related. ${ }^{6}$ Over the 6 years of data for this study, the frequency of occupational exposure claims increased from 78 in 1995 to 216 in $2001 .^{6}$ Out of the 562 exposures where follow up data were available, 41 had evidence of exposure to $\mathrm{HBV}, \mathrm{HCV}, \mathrm{HIV}$, or $\mathrm{HBV} / \mathrm{HCV}$ co-infection. ${ }^{6}$

\section{AIMS}

Dentists and other members of the dental team are at risk of exposure to blood-borne viruses. There is evidence to suggest that dentists and other members of the dental team are under-reporting occupational exposures, however, reasons for underreporting in primary dental care are unclear. ${ }^{16,17}$ Evidence to date suggests that the greater proportion of sharps injuries occur as a result of inappropriate handling and disposal of instruments. ${ }^{6,18,19}$ In addition, few studies have explored the attitudes and beliefs of primary dental care practitioners towards reporting occupational exposures in primary dental care. Given this combination of factors the aim of the study was to evaluate dentists' current reporting behaviour as well as their beliefs about reporting occupational exposures to blood and other oral fluids in Scottish dental practices.

\section{METHODS}

This study was a cross-sectional survey of a random sample of dentists working in primary dental care in Scotland. Because current practice with respect to reporting of occupational exposure incidents is likely to be practice-based, no more than one dentist per practice was invited to take part. The sample was identified from a database provided by Information Services Division (ISD) to the Dental Directorate in NHS Education for Scotland. This database collates publicly available information of the names of all dentists in Scotland providing primary care NHS dental services and their practice addresses. The random sample of dentists was generated using a two-stage simple randomisation procedure with computer generated random numbering. In stage one, a random sample of 300 dental practices (approximately one third of total dental practices in Scotland) was generated. In stage two, for practices with more than one dentist, computer generated numbers were used to select the dentist for inclusion in the sample. The gender and health board profile of the final sample of dentists was similar to the whole population sample. Based on response rates in previous surveys conducted by the authors within the same population, the sample size was determined by assuming 5\% of questionnaires would be undeliverable with a response rate from the remaining 285 questionnaires of 50\% giving an error rate of 7.5\% and confidence level of 95\%. All questionnaires were sent by first class post with a personalised letter and a freepost envelope for their return. The first mailing was conducted in April 2011 followed by a reminder mailing with a further copy of the questionnaire at 3 weeks, with a final reminder to non-responders at 6 weeks.

The questionnaire was divided into three sub-sections. Using a response set of 'yes/no/not sure', section one measured whether or not the practice in which the dentist worked had protocols or procedures for reporting occupational exposure of staff or patients to sharps injury, blood splash or saliva exposure. In addition, dentists were asked about their own occupational exposure during the previous 12 months and how any such exposures were reported.

Section two measured dentists' beliefs regarding the reporting of occupational exposures to blood or saliva using theoretical constructs from the theory of planned behaviour (TPB) that have been show to influence behaviour in general dental practice..$^{20}$ The TPB predicts that an individual is more likely to report an occupational exposure if they have high intention to do so, if they think it will be easy for them to do (high perceived behavioural control) and if they believe that doing so will result in positive outcomes. ${ }^{21}$ All beliefs were measured using seven point Likert scales where higher scores denote more positive beliefs. Attitude was measured by asking dentists to rate the importance (not at all important/very important), cost effectiveness, necessity (not at all necessary/very necessary) and practicality (not at all practical/very practical) of reporting their own or patients' occupational exposures. Dentists were also asked if they intended to report their own or their patients' occupational exposures, if they were under any pressure to do so and if they believed the costs of doing so outweighed the benefits. Perceived behavioural control was measured by asking dentists about how difficult (very difficult/not at all difficult) they think it is to report these exposures, to avoid incurring trouble by doing so and to risk assess the patient and type of exposure. Section three measured the dentist's and practice's demographics.

Before its use, the questionnaire was piloted with a small convenience sample of dentists working in primary care dental practice to assess its face validity and to gauge dentists' understanding and comprehension of the questions.

Analysis included descriptive statistics for each question. Comparisons between questions were done using Wilcoxan signed rank tests. Significance was defined as $p$ value $<0.05$. Free text responses were independently analysed and coded by two researchers. Coding was agreed and any discrepancies were resolved through discussion before themes were identified and agreed. ${ }^{22}$ 


\begin{tabular}{|c|c|c|}
\hline Demographic variable & \multicolumn{2}{|l|}{ Response } \\
\hline Gender & Female, 35\% & Male, 65\% \\
\hline Role & Principal, 67\% & Associate, 33\% \\
\hline Practice & General dental service (GDS), 96\% & Other (salaried or other), 4\% \\
\hline Vocational trainer & Yes, 22\% & No, $78 \%$ \\
\hline Employ dental hygienist/therapist & Yes, 65\% & No, $35 \%$ \\
\hline Sessions per week & \multicolumn{2}{|c|}{ Mean 8, range (minimum 3, maximum 11) standard deviation 1.8} \\
\hline Dentists per practice & \multicolumn{2}{|c|}{ Mean 3, range (minimum 1, maximum 9) standard deviation 1.6} \\
\hline Age & \multicolumn{2}{|c|}{ Mean 45 , range (minimum 23, maximum 71 ) standard deviation 10.4} \\
\hline
\end{tabular}

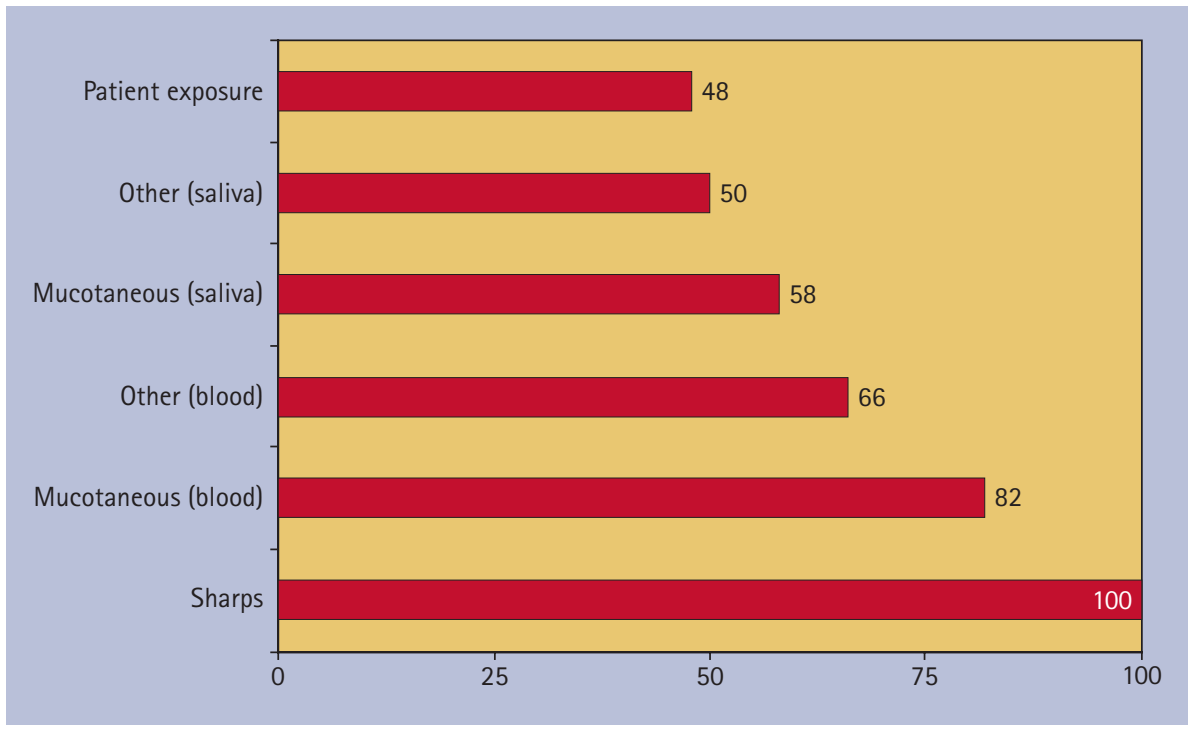

Fig. 1 Percentage of practices with protocols by exposure type

The East of Scotland Research Ethics Service considered this study in February 2011 and under the terms of Governance Arrangement for Research Ethics Committees it was advised that full ethical review was not required for this project as it was considered a service evaluation and the questionnaire used was anonymised.

\section{RESULTS}

A total of 124 surveys were returned out of the original 300. Seven dentists in the original sample had retired or moved from their practice, resulting in a valid sample of 293 dentists. The response rate for this survey was $42 \%$. Responders did not differ significantly from non-responders by available variables (gender and health board). The majority of respondents were male (65\%). Most (96\%) worked in the general dental service, and a small percentage $(4 \%)$ worked in the salaried service or identified as 'other'. Two thirds (67\%) were principals and one third (33\%) were associates. Respondents ranged in age from 23 to 71 years with an average age of 45 years. A quarter of respondents $(22 \%)$ were vocational trainers. Two thirds $(65 \%)$ of dentists said their practice had an in-house dental hygienist or dental therapist. Results are summarised in Table 1.

\section{Protocols for occupational exposure incidents}

All respondents' practices had a protocol or procedure in place for the reporting of sharps injuries incurred by members of the dental team. Protocols for specific types of exposure were not as common or familiar to respondent dentists. Protocols for mucocutaneous blood exposure (eyes, nose, etc) were present in $82 \%$ of practices. Protocols for other blood splash exposures were reported in 66\% of practices. Mucocutaneous saliva exposure protocols were reported in 58\% of practices; other mucocutaneous saliva exposure protocols were present in 50\%. Protocols for reporting patients' exposures to blood or saliva from a member of the dental team were present in $48 \%$ of practices. Results are summarised in Figure 1.

\section{Dentist occupational exposure incidents and reporting}

Over the past 12 months, the majority of dentists (84\%) did not have any exposures to patients' blood or saliva. Seventeen dentists $(14 \%)$ had one or more exposure incidents in the past year; two dentists had occupational injuries in which they were unsure whether or not they had been exposed to blood or saliva. Of the dentists with a known exposure incident, 13 had a single exposure and four had two exposures. Following exposure, six (35\%) dentists did not report the exposure to anyone, six (35\%) reported to their practice manager and local occupational health service, two $(12 \%)$ reported to their practice manager only, and the remaining three $(18 \%)$ reported to a combination of the above entities plus hospital accident and emergency.

\section{Dentists' beliefs and behaviours toward reporting occupational exposures}

Using seven point Likert scales ( 1 = strongly disagree, $7=$ strongly agree) respondents rated the importance (median $=6$ ), necessity (median $=6)$, ease $($ median $=6)$, and practicality (median $=5$ ) of reporting their own exposures. Reporting patient exposures was rated significantly higher in terms of importance (median $=7, \mathrm{z}$-score -4.44 , $\mathrm{p}$ value $<0.001$ ), necessity (median $=7, \mathrm{z}$-score $-4.17, p$ value $<0.001$ ), and practicality (median $=6$, $\mathrm{z}$-score -3.62 , $\mathrm{p}$ value $<0.001$ ). No significant difference was found for ease of reporting patient exposures compared to reporting dentist exposures (z-score -1.35, $\mathrm{p}$ value $=0.18$ ) but dentists' intention to report patient exposures (median $=7$ ) was significantly higher (z-score -3.82, $\mathrm{p}$ value $<0.001$ ) than intention to report their own exposures (median $=6$ ). Dentists were neutral when asked whether or not they felt under pressure to report exposures for themselves (median $=4$ ) and for patients (median $=4)$. Dentists were also fairly neutral in their views on whether the benefits outweighed the costs of reporting, though again expressed a significantly more positive view for patients $($ median $=4)$ than for themselves (median $=3, \mathrm{z}$-score -2.93, $\mathrm{p}$ value $=0.003$ ). Dentists did not think reporting exposures would cause their colleagues (median $=2$ ) or patients (median $=2$ ) to lose faith in their competence.

When reporting exposures in practice, respondents were neutral about their ability to find the necessary time for reporting; to assess the source patient's risk of communicable disease; or to assess risk of disease transmission given the type of exposure. Reporting was perceived as fairly 
easy (median $=5)$. Finding information on how to report exposure was not perceived as difficult (median $=6$ ). Respondents were confident (median $=6$ ) they could successfully negotiate the reporting system.

The number of dentists (17) who experienced an occupational exposure (OE) was too small to allow meaningful comparison between those who reported the OE (12) and those who did not (5). Median responses were the same for both groups in terms of their intention to report their own exposure (median $=5$ ) or a patient's exposure (median $=7$ ). Non-reporting dentists had lower median scores related to ease (nonreporting median $=3$; reporting median $=5$ ) and practicality (non-reporting median $=3$, reporting median $=4$ ) of reporting their own exposure. Non-reporting dentists also scored lower in terms of being able to find the correct reporting procedure (nonreporting median $=4$, reporting median $=6$ ) and feeling they could protect patient confidentiality in reporting (non-reporting median $=4$, reporting median $=6.5$ ) .

\section{Free-text responses}

Dentists were invited to leave free-text responses in relation to any past experience of occupational exposure or any aspect of the questionnaire. A total of 38 respondents left comments. Thematic analysis of comments identified three emergent themes: risk assessment, risk management and concerns about occupational health services.

Free text relating to risk assessment included past experiences and current attitudes toward determining risk from an exposure. Dentists discussed performing informal assessments, judging factors such as age, gender, and exposure route. A common view was that 'there is a world of difference infectivity-wise between a needle stick from an IV drug user and a cough or sneeze from a 60-year-old'. Risk management focused on practice efforts to prevent and minimise occupational exposure such as use of personal protective equipment, safe technique, and clear protocols. Minimising risk, particularly in a dental setting ' $w$ here it is impossible to detect or report every single exposure to saliva' was seen as 'essential'.

Concerns about occupational health related largely to confusion about how the service operates, the purpose of reporting exposures and the sense that occupational health does not offer any services or advice beyond what dentists can provide for themselves.

\section{DISCUSSION}

This study evaluated how dentists in Scotland think about and manage occupational exposures to blood and other oral fluids. Non-responders did not differ significantly from responders by available demographics (gender and health board) and our response rate was consistent with similar studies. ${ }^{17,23}$ The percentage of dentists with an occupational exposure (14\%) in the past 12 months was similar to other studies, ${ }^{17}$ as was the frequency of reporting (65\%). ${ }^{4}$ While all respondent's practices had protocols in place for the reporting of sharps injuries incurred by dental staff, and most practices (82\%) had a protocol for mucocutaneous blood splash, half of all respondent practices were missing some type of reporting protocol (for example, mucocutaneous saliva) for staff exposure. This widespread absence of reporting protocols may represent a gap in health and safety within the dental service. Potential gaps in patient safety are even more widespread with the absence of protocols or procedures for patient exposure in over half (52\%) of respondent practices. Issues related to patient safety have been highlighted in other studies. A study by Thusu et al. ${ }^{24}$ of over 2,000 "patient safety incidences', which included radiography errors, adverse reactions and medical emergencies, showed that injuries sustained during treatments occurred in 10\% of cases. ${ }^{24}$ The most common types of injuries sustained were lacerations, sharps injuries and burns to the lips, tongue and buccal mucosa. These types of incidents represent a potential cross-infection hazard to the patient and given that less than half of our respondents had protocols in place to deal with such occurrences, this suggests further research is needed on this topic, along with clinician education and awareness.

This study also explored dentists' beliefs and behaviours toward reporting occupational exposures. The majority of respondents did not find reporting difficult and felt reporting is very important, necessary, and something they intend to do although they were neutral about the balance between the costs and benefits of reporting. Beliefs about reporting patient exposures were often significantly more positive than attitudes toward reporting dentist exposures. Free text responses, reiterated differential behaviours and attitudes toward reporting dentists' versus patients' exposures.

Overall, variation in the types of protocols present in clinics and the neutrality of dentists when comparing the costs and benefits of reporting stand in contrast to dentists' attitudes and intention to report. Based on these initial findings, it appears that while some dentists appear complacent about risk and/or identified barriers within the reporting system, the majority of dentists have some degree of motivation and perceived capability around reporting. This motivation and capability does not always translate into action (for example, protocols for patient exposure), which suggests future interventions such as action planning may be indicated.

This study is an initial examination of current practice and attitudes toward occupational exposure in the dental primary care setting in Scotland. As such, there are several limitations. First, although our response rate was similar to other questionnaire-based studies in primary dental care, there is a risk of response bias. Deliberate efforts to maximise response rates $^{25}$ were made (for example, first class outward mailing, follow up reminders and duplicate questionnaires, assured confidentiality, colour printing, university sponsorship) and available demographics did not differ between responders and nonresponders. Despite a low response rate, the proportion of dentists reporting an $\mathrm{OE}$ in this study was similar to other studies. A higher response rate would help reduce the risk of response bias; low-cost, effective strategies ${ }^{25}$ to use for similar surveys in future could include use of a teaser on the envelope or non-monetary incentives (for example, continuing professional development hours).

Although it is recognised that participation in surveys by healthcare professionals has been in decline perhaps owing to a lack of time or perceived low value of studies, ${ }^{26}$ the reasons behind our low participation rate remains unclear. Second, because of the small number of dentists who had an exposure in the past 12 months, we could not make meaningful comparisons to dentists who were not exposed. Given the response rates in this population, using a larger sample size in future could allow for enough responses to enable meaningful comparison between dentists (for example, those with an OE and those without). Third, this study focused only on dentists and did not extend to other registrant groups such as dental nurses. Available evidence strongly suggests that attitudes and behaviour vary across registrant groups and by experience (years in practice) for both exposure and reporting. ${ }^{7,14,18,19,27}$ Considering different registrant groups and the length of time registrants have been in practice or qualified would be useful in future studies.

\section{CONCLUSION}

This study suggests that while dentists generally have positive beliefs about reporting occupational exposures, there are gaps in practice. Future exploration of this topic should include other members 
of the dental team (for example, dental nurses) to determine whether frequency of occupational exposure incidents, reporting behaviour, beliefs and barriers are similar or different across registrant groups. Subsequent work could then be undertaken to clearly identify the barriers to reporting in the dental team as a whole and thereby inform future interventions (for example, training regarding risks, reporting guidance, changes within occupational health services) to improve reporting behaviours.

1. Smith A J, Cameron S O, Bagg J, Kennedy D. Management of needlestick injuries in genera dental practice. Br Dent J 2001; 190: 645-650.

2. Public Health Laboratory Service AIDS, STD Centre and Collaborators. Occupational transmission of HIV. Summary of published reports. London: PHLS AIDS and STD Centre at the Communicable Disease Surveillance Centre and Collaborators, 1999.

3. Department of Health. Health Clearance for tuberculosis, hepatitis $B$, hepatitis $C$ and HIV: new healthcare workers. London: $\mathrm{DH}, 2007$.

4. Wicker S, Rabenau H F. Occupational exposures to bloodborne viruses among German dental professionals and students in a clinical setting. Int Arch Occup Environ Health 2010; 83: 77-83.

5. Leggat P A, Kedjarune U, Smith D R. Occupational health problems in modern dentistry: a review. Ind Health 2007; 45: 611-621.

6. Shah S M, Merchant A T, Dosman J A. Percutaneous injuries among dental professionals in Washington
State. BMC Public Health 2006; 6: 269.

7. Stewardson D A, McHugh S, Palenik C J, Burke F J T. Occupational exposures occurring among dental assistants in a UK dental school. Prim Dent Care 2003; 10: 23-26

8. Department of Health. Getting ahead of the curve: a strategy for combating infectious diseases (including other aspects of health protection). A report by the Chief Medical Officer. London: DH, 2002

9. Health Protection Agency. Hepatitis C in the UK: 2013 report. London: HPA, 2013.

10. Health Protection Agency. HIV in the United Kingdom: 2012 report. London: HPA, 2012.

11. Department of Health. HIV post-exposure prophylaxis: guidance from the UK Chief Medical Officers' Expert Advisory Group on AIDS. London: $\mathrm{DH}, 2008$.

12. Kennedy I, Williams S. Occupational exposure to HIV and post-exposure prophylaxis in healthcare workers. Occup Med 2000; 50: 387-391.

13. Health Protection Agency. Eye of the needle: United Kingdom surveillance of significant occupational exposures to bloodborne viruses in healthcare workers. London: HPA, 2012.

14. Stewardson D A, Burke F J, Elkhazindar M M et al The incidence of occupational exposures among students in four UK dental schools. Int Dent J 2004; 54: 26-32.

15. Younai FS, Murphy D C, Kotelchuck D. Occupational exposures to blood in a dental teaching environment: results of a ten-year surveillance study. J Dent Educ 2001; 65: 436-448.

16. Nagao $M$, linuma $Y$, Igawa J et al. Accidental exposures to blood and body fluid in the operation room and the issue of underreporting. Am J Infect Contro/ 2009; 37: 541-544

17. McDonnell C. An audit on reporting and management of occupational exposures to blood and body fluids in the Irish dental setting. MFOMI Dissertation. Dublin: Royal College of Physicians of Ireland Library, 2008.

18. Cuny $E$, Hoover T E, Kirk J S. Underreporting of bloodborne exposures in a dental school clinic. J Dent Educ 2011; 75: 544-548.

19. Myers J E, Myers R, Wheat M E, Yin M T. Dental students and bloodborne pathogens: occupationa exposures, knowledge, and attitudes. J Dent Educ 2012; 76: 479-486.

20. Bonetti D, Young L, Black I, Cassie H, Ramsay C R, Clarkson J. Can't do it, won't do it! Developing a theoretically framed intervention to encourage better decontamination practice in Scottish dental practices. Implement Sci 2009; 4: 31.

21. Ajzen I. The theory of planned behaviour. Organ Behav Hum Decis Process 1991; 50: 179-211.

22. Ryan $G$, Bernard H R. Techniques to identify themes. Field Methods 2003: 15: 85-109.

23. Lazenby M G, Anderud J, Whitley S P. Blood-borne viruses: are we taking them seriously? A survey of UK oral and maxillofacial surgeons. Br J Oral Maxillofac Surg 2011; 49: 400-403.

24. Thusu S, Panesar S, Bedi R. Patient safety in dentistry - state of play as revealed by a national database of errors. Br Dent J 2012; 213: E3.

25. Richards D. How to increase response rates to postal questionnaires. Evid Based Dent 2007; 8: 53-54.

26. VanGeest J B, Johnson T P, Welch V L. Methodologies for improving response rates in surveys of physicians- A systematic review. Eval Health Prof 2007; 30: 303-321.

27. Winchester S A, Tomkins S, Cliffe S, Batty L, Ncube $F$, Zuckerman M. Healthcare workers' perceptions of occupational exposure to blood-borne viruses and reporting barriers: a questionnaire-based study. J Hosp Infect 2012; 82: 36-39. 